\title{
斜圆柱结构的新型微细球端铣刀的设计与制造*
}

\author{
方泽平 刘志兵 何理论 王西涁 焦 黎 \\ (北京理工大学先进加工技术国防重点学科实验室 北京 100081)
}

\begin{abstract}
摘要: 针对微细切削刀具的特点与应用需求, 设计一种斜圆柱结构的新型微细球端铣刀, 将铣刀球端刀刀复杂的空间曲线转 化为易加工的平面曲线。根据所设计铣刀的几何结构特征, 从制造工艺方面进行刀具结构的调整, 分析刀具的刀磨成形原理, 并在微细刀具数控刀磨机上完成该刀具的制作。通过与传统螺旋槽球端铣刀和椭圆柱刀型球端铁刀的切削性能对比试验, 研 究所设计刀具的切削性能。试验结果表明, 所设计的微细球端立铣刀在显著降低刀具制备难度的同时, 具有较高的切削刃强 度, 能够满足硬脆性材料的微细切削要求。
\end{abstract}

关键词: 微细立铣刀; 刀具设计; 刀具刀磨; 切削性能

中图分类号: TG7

\section{Design and Manufacture of a New Micro Ball End Milling with Helical Cylindrical Structure}

\author{
FANG Zeping LIU Zhibing HE Lilun WANG Xibin JIAO Li \\ (Key Laboratory of Fundamental Science for Advanced Machining, Beijing Institute of Technology, \\ Beijing 100081)
}

\begin{abstract}
According to the characteristics of micro cutting tools and their application requirement, a new design method of micro ball end mill with helical cylindrical structure is proposed. It changes the cutting edge of ball end milling from the complex space curve into a plane curve. Based on the geometric structure characteristics of the new designed micro ball end mill, the cutting tool structure is optimized with respect to the manufacturing process. Through the analysis of the principle of tool grinding process, the designed tool is fabricated in micro NC grinder. The cutting performance of the new designed tool is investigated through cutting experiment compared to the cutting performance of traditional spiral groove ball end milling and elliptical ball end mill. The results indicate that the designed micro ball end mill can achieve higher cutting edge strength, which can meet the micro cutting requirement for hard brittle materials. In the meantime, the complexity of cutting tool preparation can decrease dramatically.
\end{abstract}

Key words: micro end mill; tool design; tool grinding; cutting performance

\section{0 前言}

微细切削刀具不是传统切削刀具在整体尺度 或局部特征尺度上的简单缩小, 而是针对微细切削 的特点和加工机理, 面向多种工程材料微小零件或 结构具体加工需求的一类特种切削刀具。针对微细 切削刀具特征尺寸小、加工难度大的特点 ${ }^{[1-3]}$, 近年 来, 许多学者开发出具有简单异形结构的新型微细 立铣刀。 $\mathrm{FANG}$ 等 ${ }^{[4]}$ 设计了一系列 D 形和三角形刀 面的立铣刀; OSAMU 等 ${ }^{[5]}$ 针对直径 $20 \mu \mathrm{m}$ 的微细

* 国家自然科学基金资助项目(50935001，51375055)。20141109 收到初 稿, 20150630 收到修改稿
立铣刀, 制作了不同刀具轮廓的微细立铣刀; CHENG 等 ${ }^{[6-8]}$ 考虑了刀具侧面和底部与工件的不必 要接触, 设计了六角形微细立铣刀; KAZUO 等 ${ }^{[9]}$ 设计了八边形的直刀铣刀结构; FLEISCHER 等 ${ }^{[10]}$ 设计了单切削刃的立铣刀; MASAHIKO 等 ${ }^{[11]}$ 设计 了简化刀型的球端微细铣刀。这些微细立铣刀切削 刃多为直刃, 以降低制备难度。然而, 无螺旋角的 设计导致这类刀具的加工性能不理想。JAN 等 ${ }^{[12]}$ 设计了螺旋角可变的单刃微细立铣刀, 有效抑制了 毛刺的生成, 但螺旋角的引入又导致刀具加工难度 的增加。

本文提出了一种新型的微细立铣刀设计方案, 将铣刀球端刀刃复杂的空间曲线转化为平面曲线, 
既易于制备又保持了 $\mathrm{S}$ 形切削刃冲击较小的优点, 同时具有较高的切削刃强度。

\section{1 新型微细球端铣刀几何结构设计}

新型铣刀采用椭圆柱刀球端铣刀 ${ }^{[13-14]}$ 的设计 方法, 切削刃部位无螺旋槽, 切削刃为一个平面与 一个半球面相交的曲线(图 1a)。相比于传统螺旋式 球端铣刀, 此类铣刀几何结构更为简单, 其球端部 与切削刃部分皆无螺旋槽, 刀具磨削较容易, 几何 形状易于保证，同时刀刃曲线具有一定刃倾角(图 $1 b)$, 具备逐渐切入、冲击较小的优点。

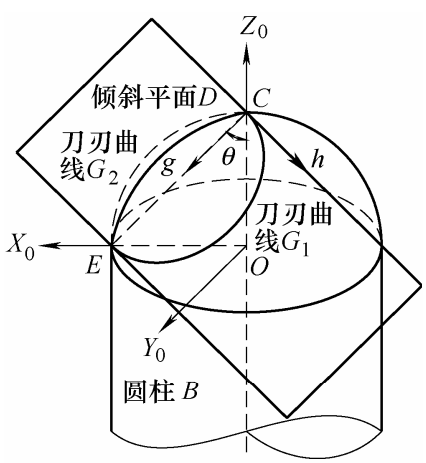

(a) 刀刃曲线的几何构成

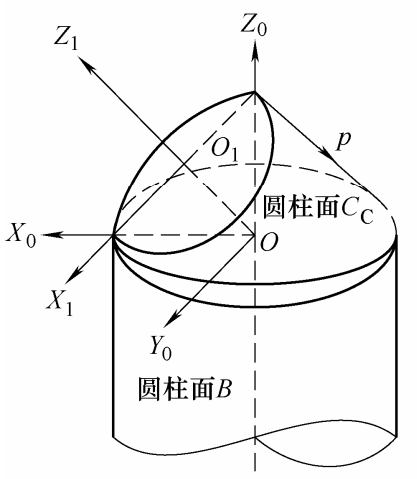

(b) 新型球端铣刀的切削刃

图 1 新型微细球端铣刀的设计方案

不同于已有的椭圆柱刃球端铣刀, 新型铣刀沿 刃口曲线向垂直倾斜面方向挤伸获得刀刃柱面(图 1b), 一方面增大刀刃楔角增强刀具切削刃强度, 同 时减小刀头结构重心偏移降低刀具的动不平衡量, 另一方面, 圆柱形轮廓的加工与精度更易于保证, 可有效降低刀具加工难度提高加工质量。

若铣刀端部的半球面 $S$ 半径为 $R$, 在图 1 所示 的坐标系中可表示为

$$
\boldsymbol{S}=\left(\begin{array}{lll}
R \cos \eta \cos \psi & R \cos \eta \sin \psi \quad R \sin \eta
\end{array}\right.
$$

式中, $\eta$ 和 $\psi$ 分别为空间极坐标系下球面 $S$ 上一点 的高低角和方位角。
相交的倾斜平面 $D$ 可由 $X_{0} Y_{0}$ 平面对 $Z_{0}$ 轴平移 $R$ 距离, 再对平移后的 $Y_{0}$ 轴旋转 $(\pi / 2-\theta)$ 角获得, 若将平移旋转前的 $X_{0} Y_{0}$ 平面表示为 $\left(\begin{array}{llll}g & h & 0 & 1\end{array}\right)^{\mathrm{T}}$, 则

$$
\boldsymbol{D}=\operatorname{Tra}(Z, R) \operatorname{Rot}\left(Y, \frac{\pi}{2}-\theta\right)\left[\begin{array}{l}
g \\
h \\
0 \\
1
\end{array}\right]=\left[\begin{array}{c}
g \sin \theta \\
h \\
-g \cos \theta+R \\
1
\end{array}\right]
$$

式中, $\operatorname{Tra}(Z, R)$ 为沿 $Z$ 轴的平移算子, $\operatorname{Rot}\left(Y, \frac{\pi}{2}-\theta\right)$ 为绕 $Y$ 轴的旋转算子。

刃口曲线为半球面 $S$ 和倾斜面 $D$ 相交形成, 可 得

$$
h= \pm \sqrt{-g^{2}+2 R g \cos \theta} \quad g \in(0, \quad R \cos \theta)
$$

则铣刀球端刃口曲线可记为

$$
\boldsymbol{G}_{i}=\left(\begin{array}{c}
G_{x i} \\
G_{y i} \\
G_{z i} \\
1
\end{array}\right)=\left(\begin{array}{c}
g \sin \theta \\
\pm \sqrt{-g^{2}+2 R g \cos \theta} \\
-g \cos \theta+R \\
1
\end{array}\right)
$$

式中， $\boldsymbol{G}_{i}$ 中 $i$ 表示第 $i$ 个刀刃，正负号分别对应第 一和第二刀刃曲线(下文相同)。

沿刃口曲线向垂直倾斜面方向挤伸获得铣刀 的刀刃柱面 $\boldsymbol{C}_{c}$, 设挤伸长度为 $p$, 则

$\boldsymbol{C}_{c}=\operatorname{Tra}(-p \cos \theta, 0,-p \sin \theta)\left(\begin{array}{c}G_{x i} \\ G_{y i} \\ G_{z i} \\ 1\end{array}\right)=\left(\begin{array}{c}G_{x i}-p \cos \theta \\ G_{y i} \\ G_{z i}-p \sin \theta \\ 1\end{array}\right)$

式中, $\operatorname{Tra}(-p \cos \theta, 0,-p \sin \theta)$ 表示沿 $(-p \cos \theta, 0$, $-p \sin \theta)$ 方向的平移算子。

\section{2 新型微细球端铣刀的刃磨}

研究中采用了牧野精机的 CNS7d 微细刀具数 控刃磨机, 机床运动机构如图 2 所示。新型微细球 端铣刀的切削刃部分为偏转 $45^{\circ}$ 角的圆柱体。因此, 刀具刃磨时, 刀具毛坏直接采用圆柱形毛坏, 无需 加工半球面。根据刀具切削刃的结构, 刃磨分为圆 柱面 $C_{c}$ 和平面 $D$ 两部分。文中重点针对圆柱面 $C_{c}$ 的刃磨进行分析。

在图 2 所示的工具磨床上, 刀具毛坏夹持在 $A$ 轴上, 现的运动只有绕自身轴线的旋转运动 $(A$ 轴 $)$ 和在工作台所在平面的旋转运动 $(W$ 轴 $)$ 。在砂轮刃 磨斜圆柱时, 因为圆柱与刀具毛坏的旋转中心轴 $Z_{0}$ (即工作主轴 $A$ 轴)存在 $45^{\circ}$ 的夹角, 因此, 在加工 
过程中, 斜圆柱轴线 $Z_{1}$ 将绕刀具毛坯的中心轴旋转 形成一个锥角为 $90^{\circ}$ 的圆雉面, 如图 3 所示。图中, $P_{1}$ 平面表示平行于 $Z_{1}$ 轴且距离为 $\frac{\sqrt{2}}{2} R$ 的平面, $P_{2}$ 表示砂轮外圆周在工作台平面的投影所在平面。此 时, 只需要在加工刀具旋转时保证 $P_{1}$ 平面与 $P_{2}$ 平 面的重合, 即可实现斜圆柱面的加工。

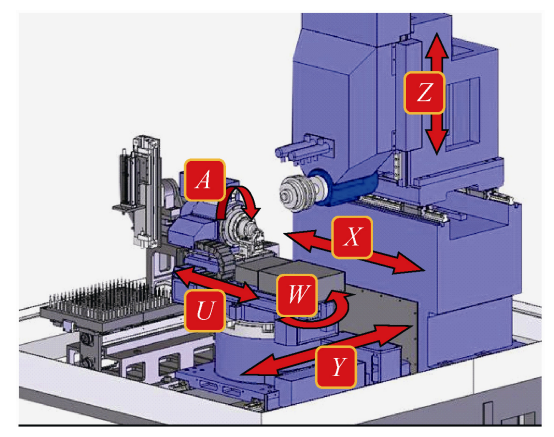

图 2 CNS7d 刀具磨床

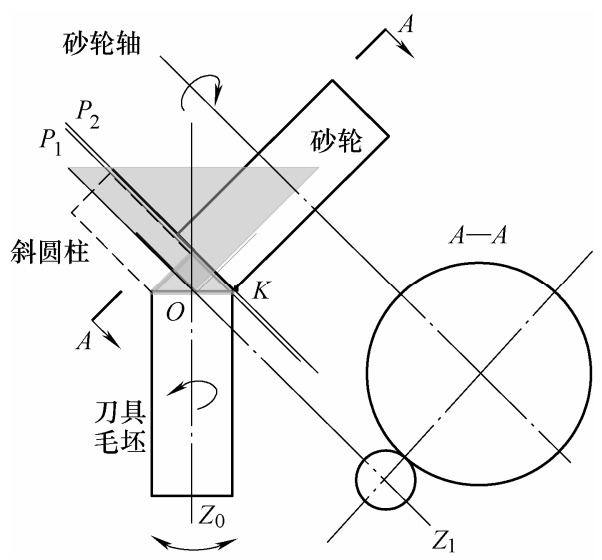

图 3 斜圆柱刃磨的相对运动

为保证 $P_{1}$ 平面与 $P_{2}$ 平面的重合, 工作主轴台 应与 $A$ 轴随动。假设加工的起始位置如图 4 所示, 此时, $A$ 轴位移为 $0^{\circ}, W$ 轴位移为 $-135^{\circ}$ 。

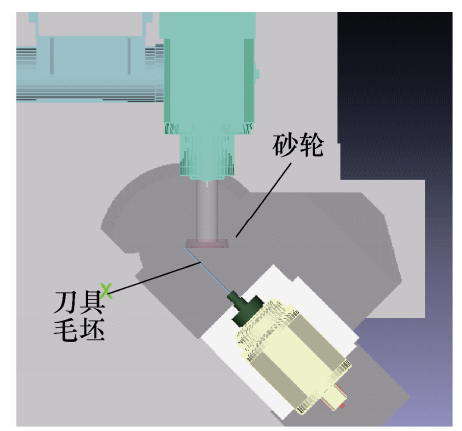

图 4 刀具刃磨的起始位置

如图 5 所示, $O M N$ 为过 $A$ 轴平行于工作台的 平面。不妨令直线 $O N$ 所在位置为加工起点, 此时, $A$ 轴的起始自转角度为 $0^{\circ}$ 。因为 $P_{1}$ 平面与 $P_{2}$ 平面 重合, 所以, $A$ 轴与砂轮轴夹角为 $45^{\circ}$ (即机床坐标 上 $W$ 轴旋转 $\left.-135^{\circ}\right)$ 。刀磨刀具时, 当刀具毛坏旋转 $\theta_{c}$ 角度时, $Z_{1}$ 轴转移到 $O P$ 位置, 其在 $O M N$ 平面 的投影为 $O Q$ 。直线 $O Q$ 与 $A$ 轴夹角 $\theta_{w}$

$$
\theta_{w}=\arctan \left(\frac{l_{O^{\prime} Q}}{l_{O O^{\prime}}}\right)
$$

式中, $l_{O^{\prime} Q} 、 l_{O O^{\prime}}$ 分别表示线段 $O^{\prime} Q$ 和 $O O^{\prime}$ 的长度。

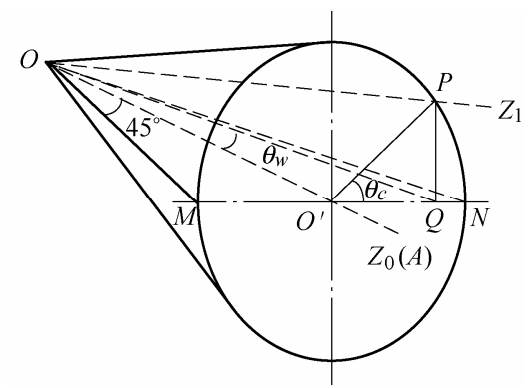

图 5 斜圆柱加工相对位置示意图

由图 5 中所示的几何关系可知

$$
\begin{aligned}
& l_{O O^{\prime}}=l_{O^{\prime} N}=l_{O^{\prime} P} \\
& l_{O^{\prime} Q}=l_{O^{\prime} P} \cdot \cos \theta_{c}
\end{aligned}
$$

式中, $l_{O^{\prime} N} 、 l_{O^{\prime} P}$ 分别表示线段 $O^{\prime} N$ 和 $O^{\prime} P$ 的长度。 因此, 有

$\theta_{w}=\arctan \left(\frac{l_{O^{\prime} Q}}{l_{O O^{\prime}}}\right)=\arctan \left(\frac{l_{O^{\prime} P} \cos \theta_{c}}{l_{O O^{\prime}}}\right)=\arctan \left(\cos \theta_{c}\right)$

因此, 当 $A$ 轴旋转 $\theta_{c}$ 角度时, 为保证 $P_{1}$ 平面 与 $P_{2}$ 平面重合, $W$ 轴应旋转相应角度, 使 $A$ 轴与 砂轮轴夹角为 $\theta_{w}$ 。

为获得所设计的刀具结构, 砂轮与刀具毛坏 $Z_{1}$ 方向还存在相对进给运动。刀具毛坏的旋转角度 $\theta_{c}$ 与砂轮 $Z_{1}$ 方向的相对进给关系如式(10)所示

$$
z_{1}=-R \cos \frac{\pi}{4} \cdot\left(1-\cos \theta_{c}\right)=-\frac{\sqrt{2}}{2} R\left(1-\cos \theta_{c}\right)
$$

式中, $R$ 为刀具刀刃回转半径。

显然, 这样的变进给速度实现难度较大, 且没 有必要。此类刀具的制备只需保证切削部分的结构, 因此砂轮 $Z_{1}$ 方向的相对进给量可采用匀速进给, 即。当磨削第一圆周刃所在半圆时, $\theta_{c} \in[0, \pi]$

$$
\begin{gathered}
z_{1}=-\left(2 R \cos \frac{\pi}{4}\right) / \pi \cdot \theta_{c} \\
v_{z_{1}}=\frac{\mathrm{d} z_{1}}{\mathrm{~d} t}=-\left(2 R \cos \frac{\pi}{4}\right) / \pi \cdot \omega
\end{gathered}
$$

当磨削第二圆周刃所在半圆时, $\theta_{c} \in[\pi, 2 \pi]$

$$
\begin{gathered}
z_{1}=-2 R \cos \frac{\pi}{4}+\left(2 R \cos \frac{\pi}{4}\right) / \pi \cdot\left(\theta_{c}-\pi\right)= \\
\left(2 R \cos \frac{\pi}{4}\right) / \pi \cdot\left(\theta_{c}-2 \pi\right)
\end{gathered}
$$




$$
v_{z_{1}}=\frac{\mathrm{d} z_{1}}{\mathrm{~d} t}=\left(2 R \cos \frac{\pi}{4}\right) / \pi \cdot \omega
$$

式中, $v_{\mathrm{z} 1 、} v_{\mathrm{z} 2}$ 分别为磨削两个刀刀时砂轮的相对进 给速度。

由于匀速进给可能导致圆柱面 $C_{c}$ 未能完全磨 削成形, 分析了刀具刀磨过程中砂轮底面绕圆柱面 $B$ 作螺旋运动所形成的运动轨迹。砂轮底面圆周 $l_{s}$ 在 $X_{1} Y_{1} Z_{1}$ 坐标系下记为

$$
\boldsymbol{l}_{s}=\left(R \cos \frac{\pi}{4}+R_{s}+R_{s} \cdot \cos \nu \quad R_{s} \cdot \sin v\right)
$$

式中, $R_{s}$ 为砂轮的半径。

根据砂轮和刀具的相对运动关系，只需要考虑 砂轮底面圆周与平面 $P_{1}$ 接触的点 $K$ (图 3)。若在图 4 所示的刀磨初始位置, 令砂轮底面与刀具平面 $D$ 平 行, 则

$$
K=\left(\begin{array}{ll}
R \cos \frac{\pi}{4} & 0
\end{array}\right)
$$

刃磨时, $K$ 点绕圆柱面 $\boldsymbol{C}_{c}$ 做螺旋运动, 在坐标 系 $X_{1} Y_{1} Z_{1}$ 形成的螺旋线 $\boldsymbol{r} *$ 如式(15)所示

$$
r^{*}=\left(\begin{array}{c}
x^{*} \\
y^{*} \\
z^{*} \\
1
\end{array}\right)=\left(\begin{array}{c}
\left(R \cos \frac{\pi}{4}\right) \cos \theta_{c} \\
\left(R \cos \frac{\pi}{4}\right) \sin \theta_{c} \\
-\frac{2 R \cos \frac{\pi}{4}}{\pi} \cdot \theta_{c} \\
1
\end{array}\right)
$$

$\boldsymbol{r}$ *在坐标系 $X_{0} Y_{0} Z_{0}$ 下可表示为

$$
r_{0}^{*}=\left(\begin{array}{c}
x * \sin \frac{\pi}{4}+z * \cos \frac{\pi}{4}+R / 2 \\
y^{*} \\
-x * \cos \frac{\pi}{4}+z * \sin \frac{\pi}{4}+R / 2 \\
1
\end{array}\right)
$$

所以, $K$ 点在坐标系 $X_{0} Y_{0} Z_{0}$ 下 $Z_{0}$ 方向的高度可表示 为

$$
z_{h}=\cos \theta_{c}-R \cdot \sqrt{1-\frac{R^{2}}{2} \sin ^{2} \theta_{c}}
$$

可解得, 当 $\sin \theta_{c}=2 / \pi$ 时, $z_{h}$ 取最大值 $z_{h \text { max }}$, 约为 $0.11 R$ 。其在坐标系 $X_{0} Y_{0} Z_{0}$ 的运动轨迹如图 5 所示。因此, 为保证刀具的切削刃部分回转结构为 完整的半球体, 应满足 $z_{h}<0$, 即砂轮的相对进给量 沿 $Z_{1}$ 方向整体下移 $z_{h \text { max }} / \sin (\pi / 4)$ 。

实际刃磨的新型微细球端铣刀的刀具整体结 构图如图 7a 所示。图 7b 为微细铣刀的刀口形貌图 像。从图 7 中可以看出, 通过所设计的刃磨方法可 获得理想的刃口形貌。

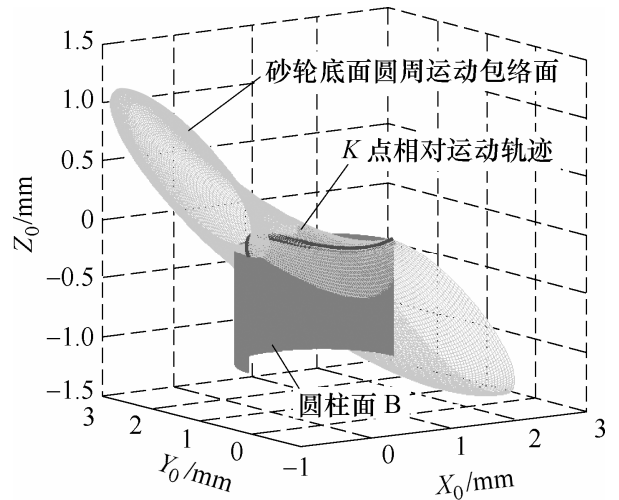

图 $6 K$ 点的相对运动轨迹

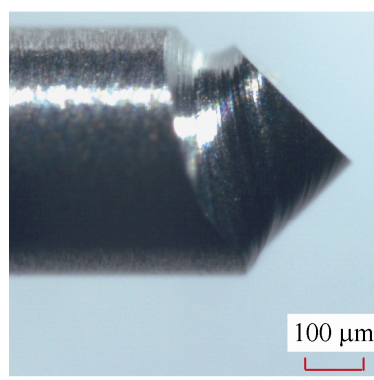

(a) 刃磨所得铁刀

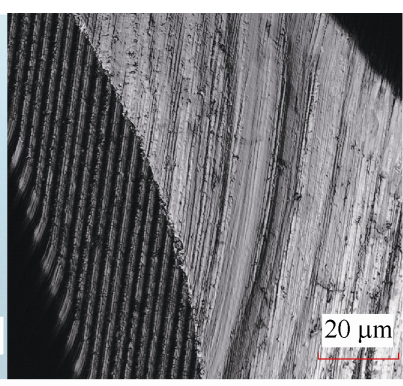

(b) 刀具局部刃口形貌
图 7 新型微细球端铣刀

\section{3 新型微细球端铣刀的切削性能}

\section{1 试验设备与条件}

为了验证所设计制造的新型微细球端铣刀的 切削性能，进行微细铣削试验。通过与传统螺旋槽 球端铣刀和椭圆柱刀球端铣刀的对比, 分析所设计 微细球端铣刀的加工特点。微细铣刀直径 $0.5 \mathrm{~mm}$, 刀具半雉角 $10^{\circ}$, 刀柄直径 $3 \mathrm{~mm}$, 刀具材料为硬质 合金 K10。选择铸铁 HT250 和 Al-6061 作为工件材 料。试验采用上海交通大学自行搭建的微细铣削平 台, 见图 8, 微细铣刀刀轴垂直于工件表面。

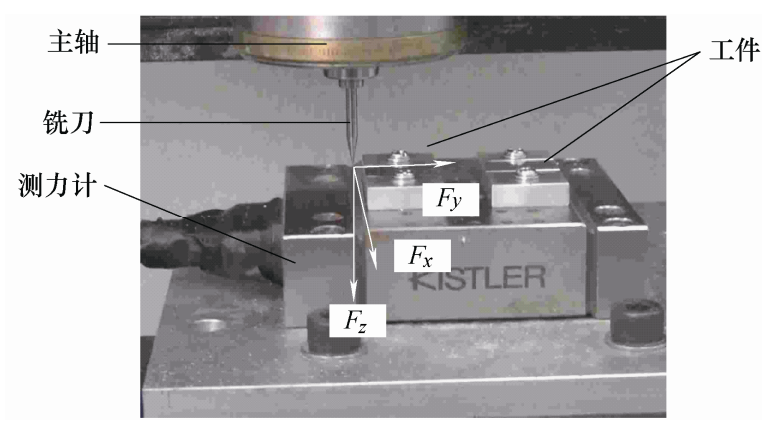

图 8 试验装置示意图

\section{2 试验结果与讨论}

\section{2 .1 铣削力}

切削 HT250 时, 设置主轴转速 $40000 \mathrm{r} / \mathrm{min}$, 进给速度 $1.5 \mu \mathrm{m} / \mathrm{r}$ ，铣削宽度 $0.02 \sim 0.08 \mathrm{~mm}$, 轴向 切削深度 $0.15 \mathrm{~mm}$ 。图 9 分别为微细铣削铸铁时测 
得 $x 、 y$ 和 $z$ 方向的力。随着铣削宽度的增加, $x$ 和 $y$ 向铣削力逐渐增大; $z$ 向上的铣削力初始较小, 随 着铣削宽度增加, 有微量增加, 主要原因是铣削加 工时刀具的磨损。在 $x$ 和 $y$ 向上, 新型微细铣刀的 切削力最大, 但在铣刀轴向 $z$ 方向新型铣刀的力反 而最小。综合考虑, 单从铣削力的大小, 新型微细 铣刀与传统螺旋槽铣刀和椭圆柱刀型铣刀基本相 当, 甚至略有优势。

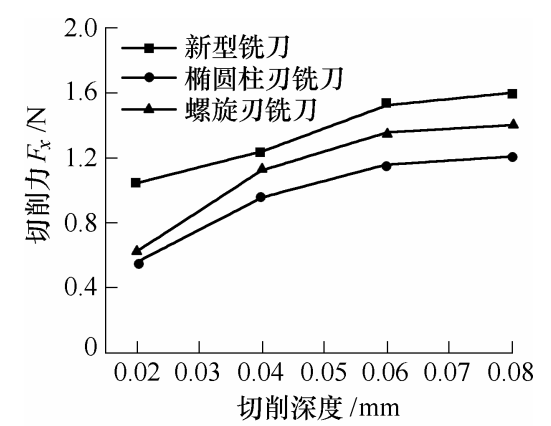

(a) $x$ 方向切削力

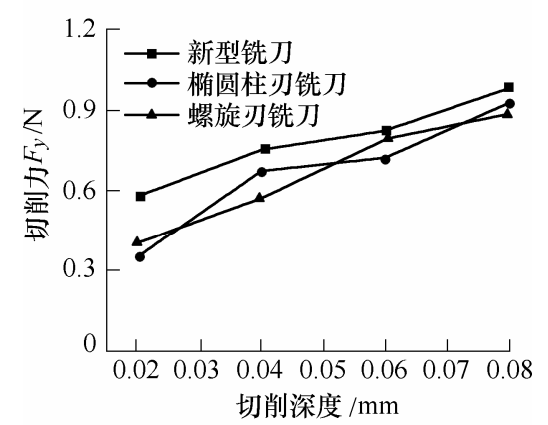

(b) $y$ 方向切削力

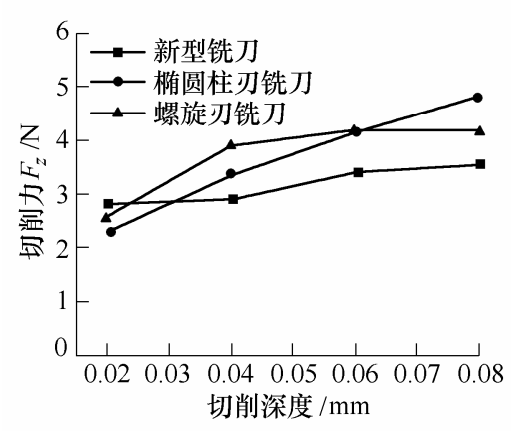

(c) $z$ 方向切削力

图 9 铣削力

\subsection{2 刀具磨破损}

通过基恩士激光显微镜观察三种不同铣刀铣 削加工后的磨破损形貌, 见图 10。相比于椭圆柱刃 型铣刀和传统螺旋槽铣刀, 新型微细球端铣刀具有 大负前角、 $90^{\circ}$ 刀刃楔角, 刀刃强度好。从刀具磨 破损形貌可知, 椭圆柱刃型铣刀和传统螺旋槽铣刀 出现崩刃现象(图 10a、10b), 而新型铣刀仅存在后 刀面磨损(图 10c)。

\subsection{3 加工质量}

在加工硬脆性材料时, 传统螺旋微细铣刀和椭 圆柱刃型铣刀有崩刃和破碎现象, 加工质量对比意

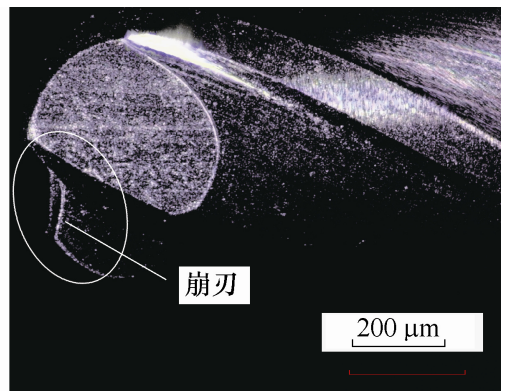

(a) 传统螺旋槽铣刀

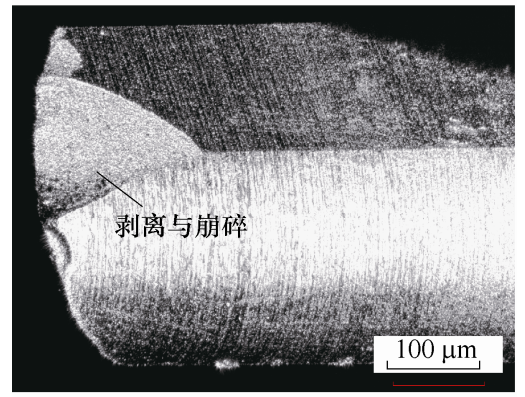

(b) 椭圆柱刃型铣刀

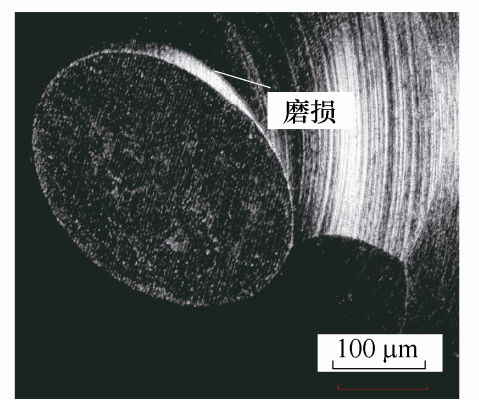

(c) 新型微细球端铣刀

图 10 刀具磨破损状态

义不明显。为了避免崩刃对加工质量的影响, 在 Al-6061 工件上铁削球面 ${ }^{[15]}$, 见图 11, 主轴转速 $20000 \mathrm{r} / \mathrm{min}$, 轴向进给速度 $1.5 \mu \mathrm{m} / \mathrm{r}$, 轴向进给深 度 $0.15 \mathrm{~mm}$ 。对比新型微细铣刀与常规铣刀的加工 形貌发现, 两柄铣刀都能获得满意的球面形状, 球 面的尺寸、形状和表面质量都符合设计要求。但新 型铣刀出口毛刺很大, 毛刺呈向外挤压的形状, 主 要原因是新型铣刀在离刀尖 $0.125 \mathrm{~mm}$ 处, 容屑空 间很小, 切屑沿切削刃向外流动形成隆起的毛刺。

通过上述对比试验可知, 新型铣刀能满足塑 性、韧性较大材料的加工要求; 特别在加工硬脆 性材料时, 由于刀具具有恒定的 $-45^{\circ}$ 大负前角和 $45^{\circ}$ 大后角, 刀刃强度高, 后刀面磨损小, 耐破损 性能强。

\section{4 结论}

根据微细切削刀具的应用特点, 在椭圆柱球端 铣刀的基础上, 提出了一种新型微细球端铣刀优化 


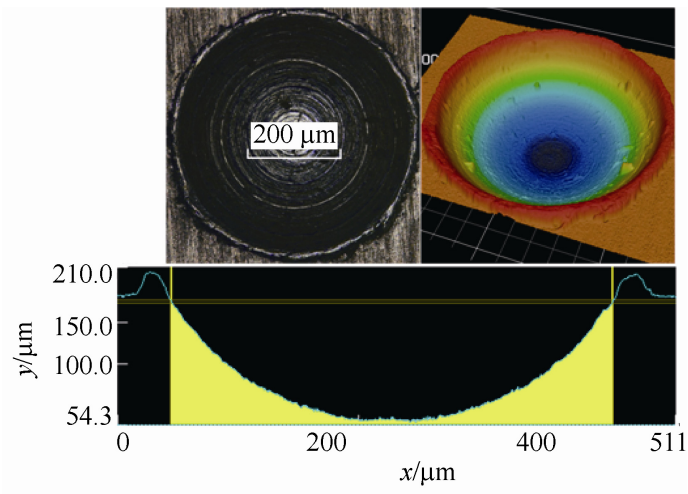

(a) 新型微细球端铣刀

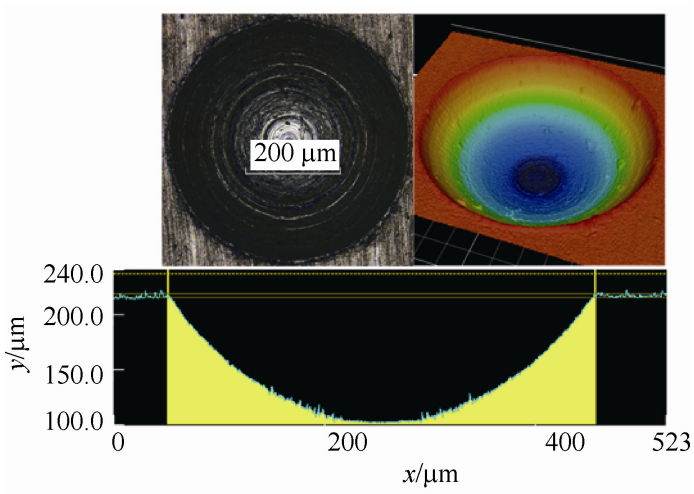

(b) 传统螺旋槽铣刀

图 11 铣钻铝合金的加工形貌

方法，在简化刀具的结构降低微细刀具制造难度的 同时增强了刀具切削部分的结构强度。文中应用微 分几何方法建立了优化的微细球端铣刀的数学模 式; 基于建立的刀具数学模型, 研究分析刀具的刃 磨工艺，获得理想的微细球端铣刀结构。通过对所 设计刀具的切削性能试验和对比试验, 验证了所设 计刀具在刀刃强度方面的优势。

\section{参 考 文 献}

[1] CHAE J, PARK S S, FREIHEIT T. Investigation of micro-cutting operations $[\mathrm{J}]$. International Journal of Machine Tools \& Manufacture, 2006, 46: 313-332.

[2] 刘志兵, 王西涁. 微细切削刀具及其相关技术研究进 展[J]. 中国机械工程, 2010，21(14): 1758-1763.

LIU Zhibing, WANG Xibin. State-of-the-art of micro cutting tool and its related technologies[J]. China Mechanical Engineering, 2010, 21(14): 1758-1763.

[3] DOMFELD D, MIN S, TAKEUCHI Y. Recent advances in mechanical micromachining $[\mathrm{J}]$. Annals of the CIRP, 2006, 55: 745-768.

[4] FANG F Z, WU H, LIU X D. Tool geometry study in micromachining $[\mathrm{J}]$. Journal of Micromechanics and Micro Engineering, 2003, 13(5): 726-731.

[5] OSAMU O , HIROMICHI O , SEUNGKI $M$, et al. Characteristics of grooving by micro end mills with various tool shapes and approach to their optimal shape[J]. Memoirs of the Faculty of Engineering, Kyushu University, 2007, 67(4): 143-151.

[6] CHENG $X$, NAKANMOTO $K$, SUGAI $M$, et al. Development of ultra-precision machining system with unique wire EDM tool fabrication system for micro/nano machining[J]. Annals of the CIRP, 2008, 57: 415-420.

[7] CHENG X, WANG Z G, NAKANMOTO K, et al. A study on the micro tooling for micro/nano milling $[\mathrm{J}]$. Int. J. Adv. Manuf. Technol., 2011， 53: 523-533.

[8] CHENG X, WANG Z G, NAKANMOTO K, et al. Design and development of PCD micro straight edge end mills for micro/nano machining of hard and brittle materials [J]. Journal of Mechanical Science and Technology, 2010, 24(11): 2261-2268.

[9] KAZUO N, KAZUTOSHI K, HITOSHI O, et al. A study on the quality of micro-machined surfaces on tungsten carbide generated by PCD micro end-milling[J]. CIRP Annals-Manufacturing Technology, 2012, 61: 567-570.

[10] FLEISCHER J, DEUCHERT M, RUHS C, et al. Design and manufacturing of micro milling tools $[\mathrm{J}]$. Microsyst. Technol., 2008, 14: 1771-1775.

[11] MASAHIKO J , ISAMU G, TAKESHI W, et al. Dvelopment of CBN ball-nosed end mill with newly designed cutting edge[J]. Journal of Materials Processing Technology, 2007, 192-193: 48-54.

[12] JAN C A, INGO G R, GUIDO M S. Manufacture and application of ultra-small micro end mills[J]. CIRP Annals - Manufacturing Technology, 2012, 61(1): 83-86.

[13] AOYAMA H, KISHINAMI T, SAITO K. Development of elliptic ball-end mill[J]. Bull. Japan Soc. Precision Engg., 1986, 20(4): 291-293.

[14] LIN P, LIN Mingfu. Geometric modelling of an elliptic ball-end mill[J]. Proc. IMechE Part B: Journal of Engineering Manufacture, 2005, 219: 87-97.

[15] 刘刚, 王亚飞, 张恒, 等. 基于分屑原理的螺旋铣孔专 用刀具研究 [J]. 机械工程学报, 2014, 50(9): 176-184. LIU Gang, WANG Yafei, ZHANG Heng, et al. Research on helical milling specialized tool based on chip-splitting principle[J]. Journal of Mechanical Engineering, 2014, 50(9): 176-184.

作者简介: 方泽平, 男, 1985 年出生, 博士研究生。主要研究方向为微 细切削刀具技术。

E-mail: zpf011@sina.com

刘志兵(通信作者), 男, 1977 年出生, 博士, 副教授。主要研究方向为 先进制造技术。

E-mail: liuzhibing@bit.edu.cn 